\title{
FE Analysis Of Runner Blade For Small Bulb Turbine
}

\author{
Mr.Manish Maisuria ${ }^{1}$, Mr. Devendra A. Patel ${ }^{2}$ \\ ${ }^{\prime}$ (Mechanical Department, CGPIT,Bardoli,India) \\ ${ }^{2}$ (Mechanical Department, CGPIT,Bardoli,India)
}

\begin{abstract}
The stress analysis of the rotor blade being the key phase of the turbomachine design requires much attention and careful determination of the blade loading to get realistic results. So due to the advantages of finite element analysis in the field of mechanical engineering it is widely used as simulation. The runner blades of the turbine are very critical part because its transfer fluid energy to convert the rotary or mechanical energy. The turbine runner blades are subjected to different load e.g. fluid or gas forces, inertia load, centrifugal forces. The primary reason of break down in turbine is the failure of blade. So, the proper blade design is require. The finite element analysis of bulb turbine is using software Creo. Initial studies focused on the modeling, meshing and physics definition of the problem. The results were analyzed for rotor and found location of several critical sections subjected to high stress concentration causing failure of rotor blade. Also found the effect of rotor speed on stress and displacement. Hence, the proper mechanical design of the turbo machine blade plays a vital role in the proper functioning of the turbo machine.
\end{abstract}

Keywords : Bulb turbine, FEA, Runner blade, stress , 13Cr4Ni

\subsection{Importance of FEA}

\section{Introduction}

The finite element method has become a powerful tool for the numerical solution of awide range of engineering problems. Applications range from deformation and stress analysis of automotive, aircraft, building, and bridge structures to field analysis of heat flux, fluid flow, magnetic flux, seepage, and other flow problems.

FEA is defined as a complex problem is divided into smaller and simpler problems that can be solved by using the existing knowledge of mechanics of materials and mathematical tools.

Modern mechanical design involves complicated shapes, sometimes made of different materials that as a whole cannot be solved by existing mathematical tools. Engineers need the FEA to evaluate their designs.

The process of dividing the model into small pieces is called meshing. The behavior of each element is well-known under all possible support and load scenarios. The finite element method uses elements with different shapes. Elements share common points called nodes.

\subsection{A General Procedure for Finite Element Analysis}

- $\quad$ Preprocessing

$>$ Define the geometric domain of the problem.

$>\quad$ Define the element type.

$>$ Define the material properties of the elements.

$>\quad$ Define the geometric properties of the elements (length, area, and the like).

$>\quad$ Define the element connectivities (mesh the model).

$>\quad$ Define the physical constraints (boundary conditions). Define the loadings.

- Solution

$>\quad$ Computes the unknown values of the primary field variable(s)

$>\quad$ Computed values are then used by back substitution to compute additional, derived variables, such as reaction forces, element stresses, and heat flow.

- $\quad$ Post-processing

Postprocessor software contains sophisticated routines used for sorting, printing, and plotting selected results from a finite element solution.

\subsection{Need of FEA in Turbo machines}

The runner blades of the turbine are very critical part because its transfer fluid energy to convert the rotary or mechanical energy. The turbine runner blades are subjected to different load e.g. fluid or gas forces, inertia load, centrifugal forces. The primary reason of break down in turbine is the failure of blade. Hence, the proper mechanical design of the turbo machine blade plays a vital role in the proper functioning of the turbo machine. 
The stress analysis of the runner blade being the key phase of the turbine design requires much attention and careful determination of the blade loading to get realistic results. The stress analysis is performed to determine the critical section as well as the stressing pattern. As the blade vibration is also a cause of failure in many cases, the determination of natural frequencies and mode shapes is also of paramount importance. The designer should take care of dynamic forces such that the frequencies of these must be away from those of the runner blades to avoid resonance, which is an undesirable phenomenon.

\section{Problem Definition \& Present Work}

The present work includes the simulation (structural analysis) of axial turbine subjected to centrifugal stresses due to rotational speed. Due to complicated shape of the blade it is not possible to calculate the stresses of rotor blade using analytical method. Hence Creo, a solid modeling \& a finite element package are used to calculate the stresses for complex geometry of rotor blades. Stress analysis can be viewed by postprocessor phase of ANSYS' 11 .

It is applied to the design of a mini hydraulic bulb turbine rotor with three blades. A streamline curvature method is used for the solution of the meridional flow and a singularity method is utilized for the blade-to-blade flow.

The three rotor blades are radially set between two coaxial cylindrical wall surfaces. The outerdiameter is $D_{0}=0.155 \mathrm{~m}$ and the inner diameter is $D_{h}=0.055 \mathrm{~m}$.

\section{Modelling And Meshing}

After the geometric modeling of the bulb turbine with the help of Creo elements 5.0, it is imported in to the ANSYS for the further process. The geometry made in the Creo parametric is converted in to the step file (.stp format) and then it is import in the ANSYS for the meshing and the boundary conditions. Geometric models of bulb turbine components are given below which is modeled in Creo software. The digital images of components \& assembly are given below.

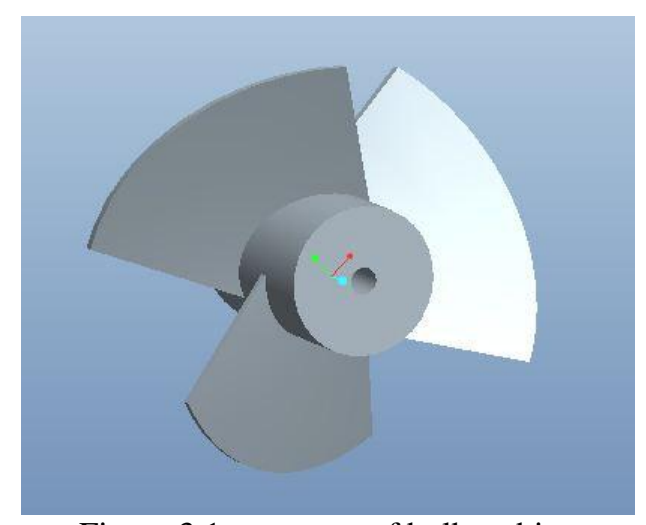

Figure 3.1 geometry of bulb turbine

Meshing is done after the geometry is successfully imported. CFD type meshing is required and for that AUTO MESH is used. In this command the ANSYS generates the meshing automatically. Following figure 3.2 shows the meshing tool in the ANSYS WORKBENCH along with the 31975 nodes and 18184 elements in meshing.

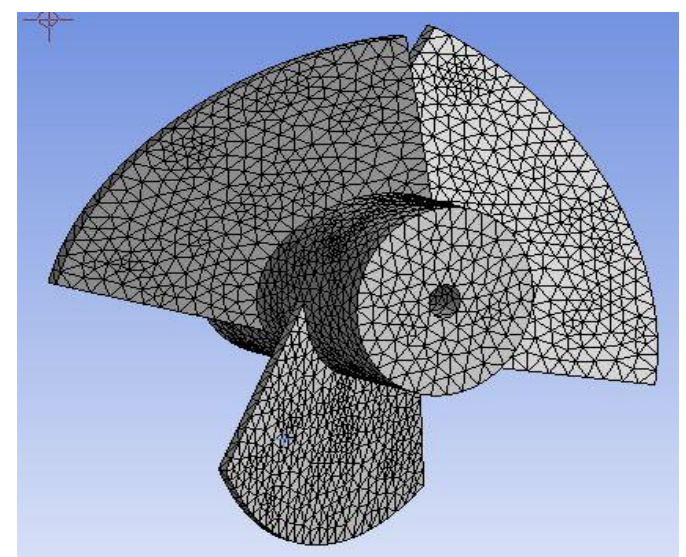

Figure3.2 FEA model with meshing 
Structural boundary conditions namely displacement restraints on the front and rear face of the shaft bore edges of rotor, while angular velocity (centrifugal load) on rotor blade model. Rotational speed of axial fan rotor $=1000$ rpm.to $5000 \mathrm{rpm}$

\begin{tabular}{l}
13 Cr4Ni \\
\hline$\square$ Structural
\end{tabular}
\begin{tabular}{|l|c|}
\hline$\square$ Young's Modulus & Add/Remove Properties \\
\hline$\square$ Poisson's Ratio & $0.06 \mathrm{e}+005 \mathrm{MPa}$ \\
\hline$\square$ Density & $7.7 \mathrm{e}-006 \mathrm{~kg} / \mathrm{mm}^{3}$ \\
\hline$\square$ Tensile Yield Strength & $580 . \mathrm{MPa}$ \\
\hline
\end{tabular}

Figure3.3 blade Material properties of $13 \mathrm{Cr} 4 \mathrm{Ni}$

IV. Analysis Results

The results obtained are presented in the form of counter maps and profiles of radial elongation, mechanical stresses on blade surface for the rotor blade of bulb turbine.

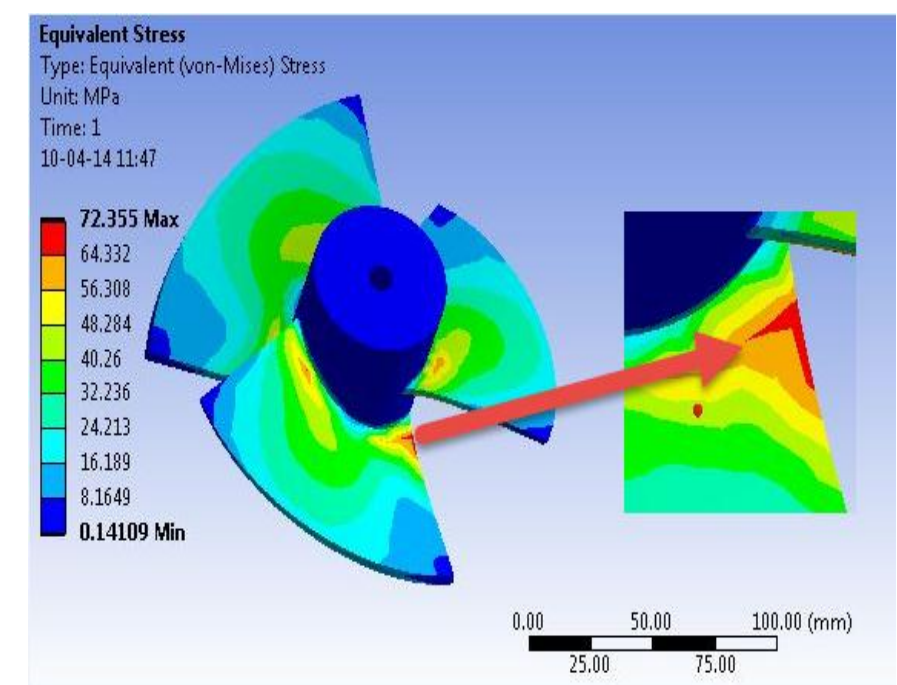

Figure 4.1 Centrifugal Stress Analysis Results of Bulb Turbine Rotor Blade

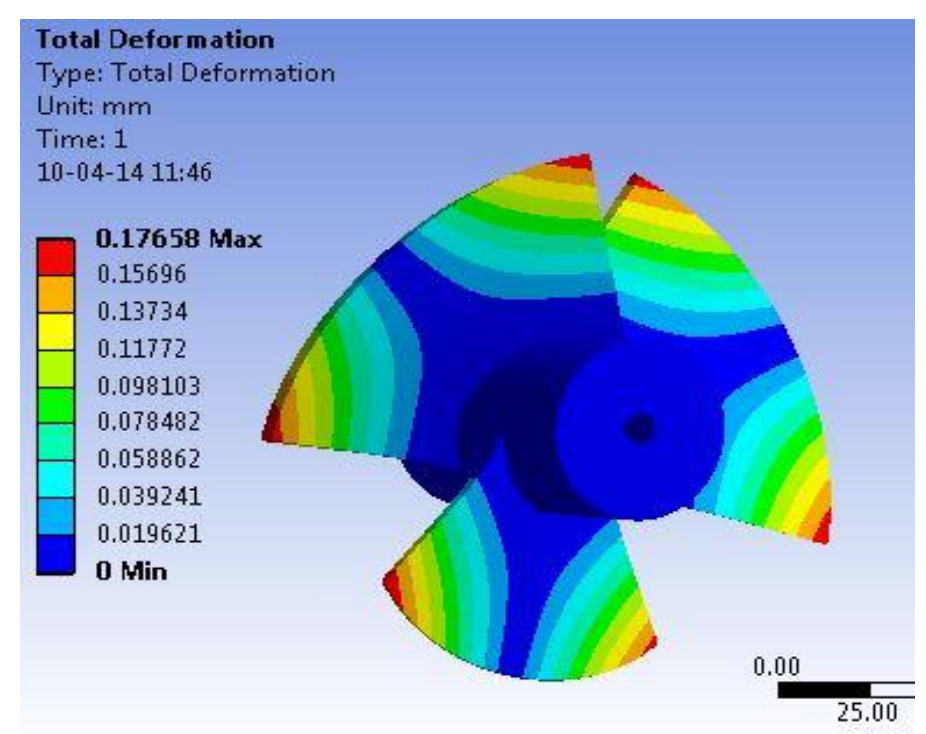

Figure 4.2 Max. Deformation analysis of Bulb turbine rotor blade

Above figure displays the stresses developed for the $13 \mathrm{Cr} 4 \mathrm{Ni}$ rotor considering a speed of $5000 \mathrm{rpm}$. Similar steps were performed by taking different material and calculating the stresses at different speed ranging from $1000 \mathrm{rpm}$ to $5000 \mathrm{rpm}$ with an increment at speed of $1000 \mathrm{rpm}$. 
Table 4.1 Max. Stresses and Max. Displacement at Various Speeds For 13Cr4Ni

\begin{tabular}{|c|c|c|}
\hline Speed (RPM) & Max. Stress(Mpa) & Max. Displacement(mm) \\
\hline 1000 & 0.272 & 0.0003 \\
\hline 2000 & 1.089 & 0.0011 \\
\hline 3000 & 2.450 & 0.0026 \\
\hline 4000 & 4.356 & 0.0046 \\
\hline 5000 & 6.806 & 0.0072 \\
\hline
\end{tabular}

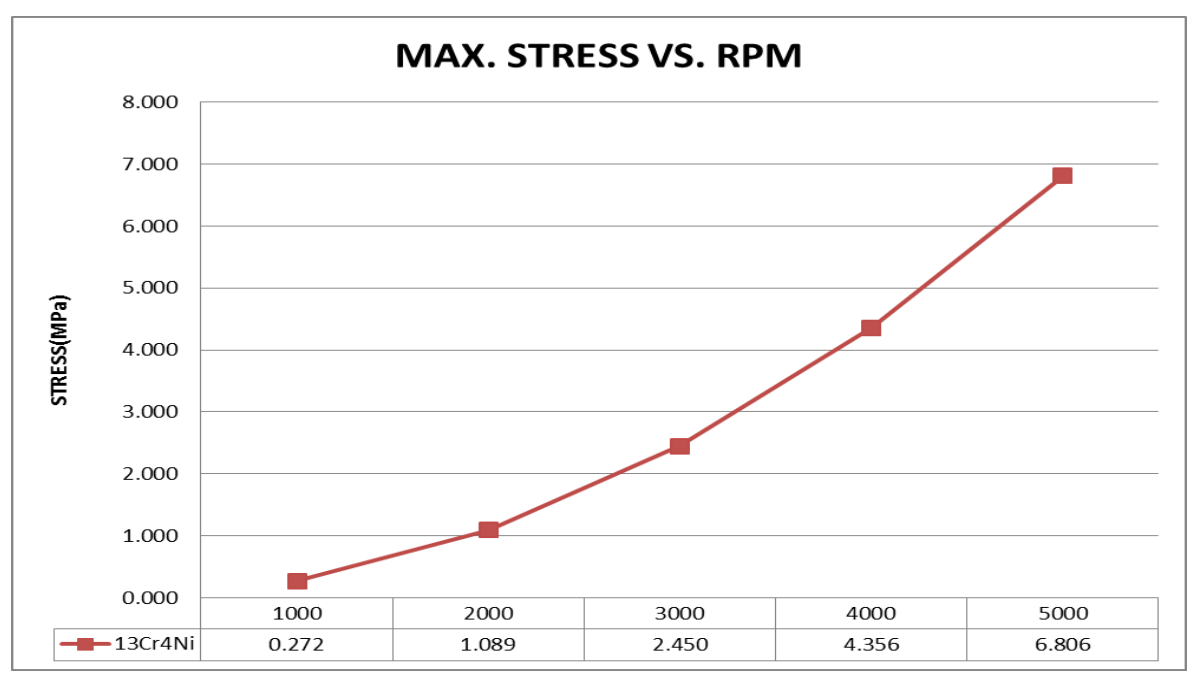

Figure 4.3 Max. Stress for 13Cr4Ni Rotor at Various Speeds

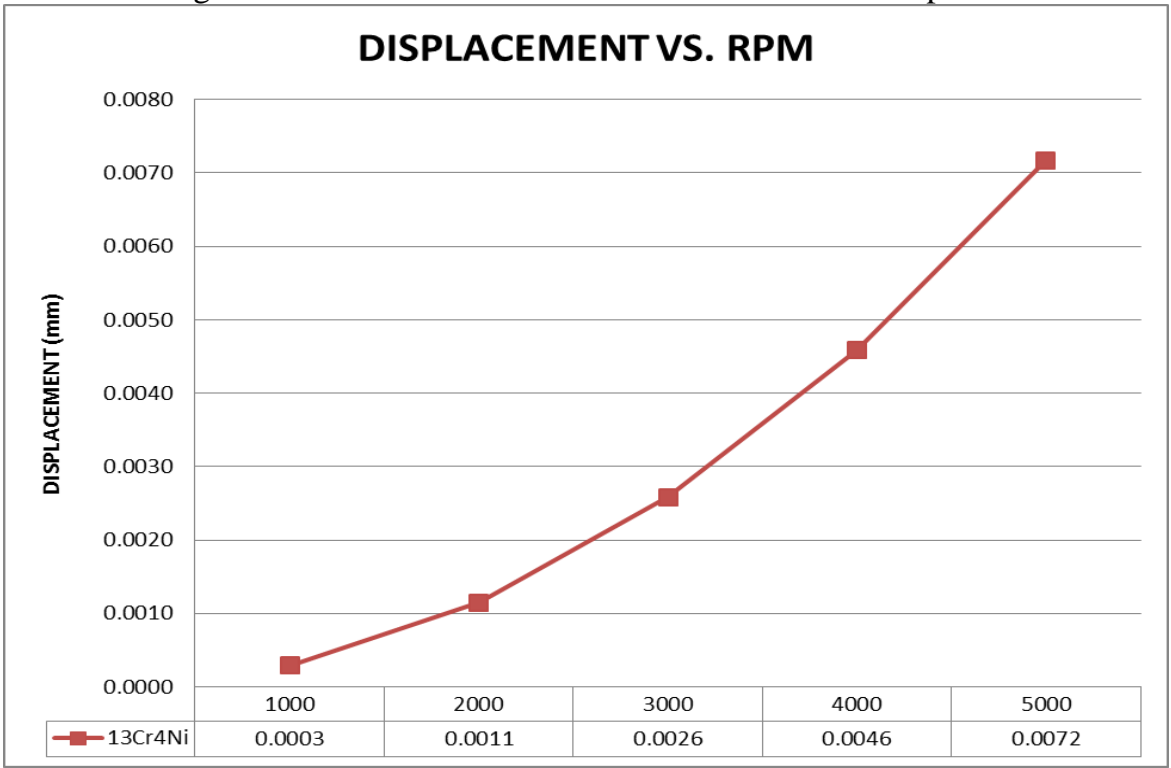

Graph 4.4 Max. Displacement for 13Cr4Ni at Various Speeds

\section{Discussion On Results}

From post processing results on graphics screen \& graphs shown above, deformation and stress results are discussed as bellows:

1. Maximum deformation is observed rotor tip section and minimum deformation at the hub.

2. Maximum stresses are observed at blade section at hub tip. The von-mises stresses are found to be in safe limit up to 5000 RPM.

3. Location of several critical sections subjected to high stress concentration causing failure of rotor blade is found at the joint of the hub and the blade.

4. Also from the graph $4.3 \& 4.4$ it is clearly seen that as the speed of rotor is increased the stress and displacement also increases.

5. Stresses produced are within safe limit. 


\section{CONCLUSION}

FE structural analysis of Bulb turbine blades using Creo software ANSYS serves as mechanical design tool. The analyzed results intimated several critical blade joint between rotor blade and hub, which are highly stressed causing blade failure so that joint requires much attention and careful determination. The von-mises stresses are found to be in safe limit up to $5000 \mathrm{rpm}$ for bulb turbine. Also as the speed of rotor is increased the stress and displacement also increases so for the high speed rotor much attention is required for material selection.

\section{References}

[1]. D Frunzăverde, R Terzi and V Şerban, "Failure analysis of a Francis turbine runner ", Earth and Environmental Science, doi:10.1088/1755-1315/12/1/012115, March 2014.

[2]. Mohammad Javed Hyder and Hafiz Laiq-ur-Rehman, "Stress Distribution of the Gas Turbine Blades," in Failure of Engineering Materials \& Structures. Code12, MED UET Taxila (2007)

[3]. Tiago de Oliveira Vale , Gustav da Costa Villar, João Carlos Menezesl,"Methodology for Structural Integrity Analysis of Gas Turbine

[4]. Blades,” J.aerosp.techno.manog., Sao Jos dos campos vol.4,No1,pp 51-59 Jan-March 2012

[5]. G.Narendranath, S.Suresh, "Thermal Analysis Of A Gas Turbine Rotor Blade By Using Ansys," (IJERA) ISSN: 2248-9622 Vol. 2, Issue 5, September-October 2012, pp.2021-2027.

[6]. Tirupathi r. Chandrupatala, "Introduction to Finite Elements in Engg." 